\title{
DETERMINAÇÃO DE PALEOCORRENTES EM CASCALHEIRAS DA FORMAÇÃO RIO CLARO (NEOCENOZÓICO)
}

\author{
NESTOR A. M. PEREIRA* e PAULO M. B. LANDIM**
}

\begin{abstract}
This study deals with the Rio Claro Formation (Neocenozoic) paleocurrent determination based on pebble main axis orientation. It was found that the particles, ellipsoidal in shape, lie with their long axis roughly parallel to flow direction and inclined downwards. The orientation data showed a southward trend for the paleocurrents, which is coincident with the present Corumbataf River drainage.
\end{abstract}

INTRODUÇÃo A Formação Rio Claro, termo estratigráfico proposto por Björnberg e Landim (1966) para sedimentos neocenozóicos malconsolidados que cobrem estratos mais antigos como, por exemplo, as Formações Estrada Nova, Pirambóia e Botucatu, na região centro-ocidental do Estado de São Paulo, possui, na sua base, cascalheiras constituídas principalmente por seixos de quartzo e quartzito e com espessura bastante variável até se tornarem uma linha de seixos, a qual tem sido referida na literatura como stone line (Penteado, 1969). Seç̧ões colunares, mostrando variações litológicas dentro da Formação Rio Claro, encontram-se em Björnberg, Landim e Meirelles Filho (1964) e uma descrição mais pormenorizada sobre a sedimentologia e estruturas sedimentares em Björnberg (1965). Outros autores que se dedicaram a esses sedimentos neocenozóicos aflorantes na Bacia do Rio Corumbataí, além dos já citados, foram Mezzalira (1964), Penteado (1968) e Fúlfaro e Suguio (1968).

No referente aos depósitos rudáceos, os estudos feitos têm se restringido às propriedades escalares dos seixos, tais como granulometria, arredondamento, esfericidade e litologia. Julgamos que o estudo de uma das propriedades vetoriais mais importantes, ou seja, a orientação das partículas poderia trazer informações úteis sobre a sedimentação desse material. O modo segundo o qual ocorre a orientação de partículas rudáceas, porém, é um assunto bastante controvertido, pois as bases hidrodinâmicas que controlam a deposição dos seixos não estão ainda perfeitamente estabelecidas. Assim sendo, como existe a hipótese, com a qual concordamos, de Björnberg e Landim (1966), que, ao definirem a Formação Rio Claro, propuseram, baseados em critérios geomorfológicos, como sentido geral de transporte, o rumo S-SW, a finalidade principal deste trabalho foi a determinação do rumo e ângulo de mergulho dos eixos maiores (a) dos seixos alongados existentes na base desses sedimentos na bacia do Rio Corumbataí, com a finalidade de, por um lado; contribuir para o estudo de paleocorrentes e, por outro, tentar determinar o sentido dos fluxos atuantes no início da sedimentação da Formação Rio Claro.

Orientação de partículas rudáceas Levando-se em consideração a influência da forma das partículas rudáceas sobre a sua orientação em depósitos sedimentares, os padrões de petrofábrica resultantes em conglomerados não se apresentam em arranjos muito complexos. Os seixos esféricos ou com um alto índice de esfericidade não mostram qualquer padrão de petrofábrica e os seixos com desigualdades em suas dimensões, isto é, prolatos ou em forma de bastão, descritos em função do seu eixo maior $a$, ou oblatos, isto é, dis-

* Bolsista da FAPESP (Processo 72/409). Endereço atual, Instituto de Pesquisas Tecnológicas, SP

**Departamento de Geologia e Mineralogia, FFCL de Rio Claro 
cóides, descritos em função do seu eixo menor $c$, podem apresentar os seguintes padrões: (a) petrofábrica isotrópica, quando não ocorre orientação preferencial; $(b)$ petrofábrica anisotrópica, quando ocorre orientação preferencial. Nesse caso a orientação pode se dar paralela ou perpendicular à direção da corrente e, ainda, com um ângulo de mergulho em relação à corrente.

Em rios onde a velocidade do fluido é alta e estável os seixos são geralmente orientados com o seu eixo maior paralelo à direção de movimentação do fluido. Johansson (1965) inclusive observou que os seixos menores de $4 \mathrm{~cm}$ mostram uma tendência maior de se alinharem segundo a direção do fluxo do que os seixos maiores. Se a velocidade da corrente, porém, é baixa ou ocorrem alternâncias entre as velocidades altas e baixas, a orientação dos eixos maiores dos seixos dar-se-á perpendicularmente à direção da corrente, como visto por Kürsten (1960), em rios intermitentes no sul da Pérsia. Em outras palavras, pode-se dizer que os seixos alongados, quando transportados em suspensão, devem, teoricamente, ter como orientação resultante os eixos maiores paralelos à direção da corrente e quando transportados por salto ou rolamento devem apresentar o eixo maior perpendicular à direção da corrente. Ê claro que, para uma interpretação correta da orientação dos seixos, há necessidade de uma perfeita avaliação de todas as condições de deposição, inclusive efeitos posteriores provocados pela própria corrente e pelos sedimentos que vierem a compor a matriz.

Além da orientação com respeito à corrente, sob certas circunstâncias, os seixos podem se depositar com o seu eixo maior mergulhando em relação ao plano de estratificação e isso é denominado imbricação. A imbricação é bem desenvolvida quando seixos alongados se apresentam bem selecionados, isto é, sem muita matriz, o que permite que as partículas fiquem em contato umas com as outras, e depositados por meio de correntes de tração.

Segundo a maioria dos autores, entre eles Potter e Pettijohn (1963), a imbricação dos seixos ocorre no sentido contrário ao da movimentação da corrente. Isso significa que, sendo encontrado um determinado vetor médio azimutal a partir de eixos maiores de seixos, a direção da corrente estaria a $180^{\circ}$, isto é, exatamente no sentido oposto.

Por outro lado, Allen (1970, pp. 80-90) afirma que partículas com formas elipsoidais jazem com o eixo maior aproximadamente paralelo à direção da corrente e imbricados com ângulos de 10 a $20^{\circ}$ no sentido de movimentação do fluido. Explica ainda que a atitude de cada partícula no espaço é a sua resposta às forças que atuam durante e logo após a deposição pelo movimento transportador. Essas forças são a de seu corpo, tendendo a mantê-lo localmente, a removedora, a qual pode ou não atuar para cima, e a de arrasto que tende a mudar as partículas de sua posição de acamamento. Admitindo que as partículas permaneçam depositadas junto a outras similares, a posição mais estável é alcançada quando as duas primeiras forças estão em equilíbrio e a última é mínima. Isso ocorre quando as partículas jazem com os eixos maiores paralelos ao fluxo e imbricados com pequenos ângulos em direção à corrente. Nesse caso, a força de arrasto é mínima e a força removedora pode mesmo ser negativa, pois os pontos de contato da partícula com outras já depositadas são diversos e localizam-se à frente de seu centro de massa.

A esse propósito, Spalletti (1972, pp. 174-176), estudando os conglomerados da região de Volcan, província de Jujuy (Argentina), concluiu que a imbricação dos seixos depende da distância de transporte, sendo que, no trecho inicial, os mesmos se inclinam contra a corrente, porém, à medida que se afastam da fonte, os seixos começam a sofrer uma inversão no arranjo deposicional e imbricam-se a favor da corrente, a partir dos trechos médio e final de transporte, com ângulo de inclinação por volta de $20^{\circ}$.

Tendo em vista essas divergências, fizemos um estudo preliminar em uma cascalheira situada sobre um terraço à margem direita do Rio Piracicaba, aflorante na estrada Pira- 
cicaba-São Pedro, e constatamos que os seixos estavam imbricados segundo a direção da corrente. Ainda como exemplo, podemos citar o trabalho de Suguio (1973a), que, estudando conglomerados e arenitos associados à fácies Uberaba (Formação Bauru) no Triângulo Mineiro, verificou que a estratificação cruzada nos arenitos indicava um sentido geral de movimentação de correntes para o sul e que os vetores médios, calculados a partir da imbricação dos seixos alongados, mostravam também valores para o sul. Tendo-se em vista a hipótese de que os seixos se imbricariam no scutido contrivio ao da corrente, no caso para o norte, esse autor estranhava a contradição. Se adotada, porém, a hipótese de que os seixos se imbricam no sentido da corrente, isto é, para o sul, a discrepância desaparece e tanto a estratificação cruzada como a imbricação dos seixos indicarão o mesmo sentido de movimentação do fluido.

Cálculo do vetor médio Medidas de direções ou rumos em ângulos são sempre obtidas em termos de uma origem arbitrária enquanto que observaçóes lineares, por outro lado, têm uma referência original definida. Desse modo, modelos estatísticos baseados em escalas numéricas lineares não podem ser aplicadas a dados angulares. Sendo as medidas em ângulos, o estudo estatístico baseia-se na chamada "distribuição circular normal" (Mises, 1918), em que se procurará verificar se a frequêencia de distribuição dos dados é uniforme por todo o círculo ou não. Não sendo uniforme, haverá uma concentração de observações em torno de uma direção preferencial, ou seja, em torno de um vetor médio. $O$ caso mais simples é o da distribuição circular unimodal, onde predomina apenas uma moda. Em casos mais complexos, há uma distribuição circular polimodal, com várias modas.

A distribuição circular normal é condicionada pelos fatores que atuam durante a sedimentação pelo fluxo de corrente, sendo as partículas geralmente orientadas de um mesmo modo específico. Flutuações na velocidade do fluido, irregularidades no fundo, efeitos por perturbações posteriores, etc. causam, porém, no acamamento, dispersóes em relação à orientação dominante. Quando colocadas em histogramas circulares ou diagramas de roseta, as observações encontradas mostram uma distribuição variada de valores angulares. Tal distribuição, que obedece a uma disposição circular, deve ser provavelmente simétrica, essencialmente unimodal, e a moda tenderá a se situar perto da verdadeira direção de movimento do fluido. $\mathrm{O}$ agrupamento das direções observadas em torno da moda deve-se ao fato de que, embora haja desvios, a corrente procura orientar as partículas segundo um mesmo rumo.

Discussões sobre o assunto são apresentadas em Reiche (1938), Pincus (1956), Steinmetz (1962), Jones (1968) e Landim, Meyer Jr. e Pereira (1974).

Resumidamente, podemos dizer que, para o somatório de dados direcionais, tendo-se medido $k$ ângulos $\alpha_{i}(i=1,2, \ldots, k)$, o $i$-ésimo ângulo tem como coordenadas $m_{i}$ e $n_{i}$,

$$
\begin{aligned}
& m_{i}=\cos \alpha_{i}, \\
& n_{i}=\operatorname{sen} \alpha_{i},
\end{aligned}
$$

as quais são utilizadas para a obtenção dos componentes do vetor médio, $M$ e $N$, segundo

$$
\begin{aligned}
& M=\Sigma \cos \alpha_{i}, \\
& N=\Sigma \operatorname{sen} \alpha_{i} .
\end{aligned}
$$

A divisão da soma algébrica dos senos $(N)$ pela soma algébrica dos co-senos $(M)$ fornecerá um valor $(V)$ tal que $\bar{\alpha}$, vetor médio ou sentido do vetor resultante, será igual a

$$
\bar{\alpha}=\operatorname{arctg}(V)
$$


a magnitude $(R)$, isto é, o comprimento do vetor médio resultante, será dada pela fórmula

$$
R=\sqrt{N^{2}+M^{2}}
$$

e a razão de consistência $(\vec{a})$ pela fórmula

$$
\bar{a}=R / k \text {. }
$$

Para verificar a hipótese nula da presença de distribuição uniforme, isto é, a não-existência de uma direção preferencial, contra a hipótese alternativa de unimodalidade, compara-se o valor $R^{2}$ encontrado com valores $R_{0}^{2}$ tabelados, em função de $k$ e segundo os níveis de significância 0,05 e 0,01 (Greewood e Durand, 1955). A hipótese nula de uniformidade será aceita se $R_{0}^{2} \geqslant R^{2}$.

Coleta de dados e metodologia usada Após localizadas diversas cascalheiras na bacia de deposição da Formação Rio Claro, foi feita uma posterior seleção, escolhendo-se dezesseis pontos de coletas que se situam nos seguintes locais:

a) pontos de 1 a 7 , ao longo do corte recentemente feito pela Ferrovias Paulista S.A. (FEPASA), entre as cidades de Rio Claro e Itirapina, para retificação da ferrovia;

b) pontos de 8 a 12, nas duas estradas que unem os municípios de Rio Claro e Ipeúna;

c) ponto 13, localizado a N-NW de Rio Claro, próximo a Jacutinga; por se tratar de uma cascalheira muito grande, esta foi submetida a coletas nos pontos $a, b, c$ e $d$, os quais foram escolhidos ao acaso e não se situam no mesmo nível;

d) pontos 14 a 16 , na estrada velha entre Rio Glaro e Ferraz.

Todas essas cascalheiras apresentam-se com matriz arenosa e estão assentadas sobre um plano irregular, em certos locais evidenciando paleocanais. A litologia predominante dos seixos é o quartzo e o quartzito, ocorrendo também pedaços de crosta de limonita de pequena espessura, tratando-se, com toda certeza, de material retransportado. $O$ tamanho dos seixos é variado, porém o eixo médio $(b)$ situa-se predominantemente entre $3 \mathrm{a} 5 \mathrm{~cm}$, chegando alguns a atingir $20 \mathrm{~cm}$. O mergulho dos eixos a não ultrapassa $20^{\circ}$. Outra característica relativa às cascalheiras é quanto a sua localização altimétrica, estando quase sempre situada entre as cotas de 580 a 600 metros de altitude.

Os seixos foram coletados, em cada local, em número superior a cem, a fim de que se pudesse fazer uma seleção prévia durante a qual foram eliminados os seixos com alto índice de esfericidade ou quebrados, pois somente interessavam os seixos alongados. Ao final, ficamos, exceto em um caso, com cem seixos.

As medidas angulares utilizadas foram tomadas em relação ao eixo maior dos seixos. Isso significa, como já dissemos, que foram selecionados indivíduos que apresentavam a relação comprimento do eixo maior $(a) /$ comprimento do eixo menor $(c)$ maior que 1,5 . Sobre a técnica de coleta de material e subseqüente manuseio dos dados, ver Suguio (1973b) ou Landim, Meyer Jr. e Pereira (1974).

Para a obtenção do vetor médio, de magnitude do vetor e da razão de consistência adotamos a metodologia recomendada por Pincus (1956), o qual apresenta uma sequiência de cálculo bastante prática e de fácil utilização.

Resultados obtidos Seguindo-se a metodologia recomendada por Pincus (1956), foram obtidos os seguintes resultados: 


\begin{tabular}{cccc}
\hline $\begin{array}{c}\text { Ponto } \\
\text { n. }\end{array}$ & $\begin{array}{c}\text { N. }^{\circ} \text { de } \\
\text { seixos }\end{array}$ & $\begin{array}{c}\text { Vetor } \\
\text { médio }\end{array}$ & $\begin{array}{c}\text { Razão de } \\
\text { consistência }(\bar{a})\end{array}$ \\
\hline 1 & 100 & $N 237$ & 0,23 \\
2 & 100 & $N 229$ & 0,26 \\
3 & 100 & $N 190$ & 0,26 \\
4 & 100 & $N 335$ & 0,19 \\
5 & 100 & $N 162$ & 0,42 \\
6 & 100 & $N 260$ & 0,30 \\
7 & 100 & $N 246$ & 0,49 \\
8 & 80 & $N 188$ & 0,24 \\
9 & 100 & $N 145$ & 0,33 \\
10 & 100 & $N 280$ & 0,26 \\
11 & 100 & $N 120$ & 0,18 \\
12 & 100 & $N 142$ & 0,40 \\
$13 a$ & 100 & $N 194$ & 0,48 \\
$13 b$ & 100 & $N 122$ & 0,22 \\
$13 c$ & 100 & $N 195$ & 0,37 \\
$13 d$ & 100 & $N 240$ & 0,37 \\
14 & 100 & $N 248$ & 0,56 \\
15 & 100 & $N 238$ & 0,25 \\
16 & 100 & $N 255$ & 0,43 \\
\hline Total geral & & $N 213$ & 0,60 \\
\hline
\end{tabular}

Esses valores direcionais foram lançados em mapa (Fig. 1), sendo o comprimento das setas proporcional à respectiva razão de consistência.

$O$ resultado geral, levando-se em conta os dezenove vetores médios locais, fornece um vetor médio resultante $N 213 \mathrm{com}$ a razão de consistência igual a 0,60 .

Interpretaçăo dos resultados A soma geral dos vetores médios obtidos pelo estudo dos eixos maiores de seixos alongados coletados em diversas cascalheiras da Formação Rio Claro mostra um valor de N213. Esse valor, adotada a hipótese de que os seixos alongados se orientam segundo o mesmo sentido de movimentação do fluido, indica que essas cascalheiras foram depositadas pelas correntes que se dirigiam de NNE para SSW. Observando, na Fig. 1, qual a disposição da drenagem do alto e médio Corumbatal verificamos que, a grosso modo, o sentido geral dessa drenagem é NNE para SSW. Ocorre, portanto, uma coincidência entre os vetores médios encontrados e o rumo geral da atual drenagem do Rio Corumbataí. Isso está de acordo com Björnberg e Landim (1966), que, como dito inicialmente ao definirem a Formação Rio Claro, propuseram o rumo geral sul que teria seguido os fluxos dominantes durante a sua sedimentação, rumo esse que é concordante com a atual drenagem (Fig. 2).

Concluindo, podemos afirmar, baseados nos resultados obtidos, que (a), no presente estudo, os seixos alongados orientam-se com seus eixos maiores segundo a direção da corrente e mergulhando no sentido da mesma; e (b) as cascalheiras aqui estudadas representam terraços ligados a uma drenagem pré-atual, a qual se dispunha com uma orientação semelhante a que existe atualmente na bacia do Rio Corumbataí. 


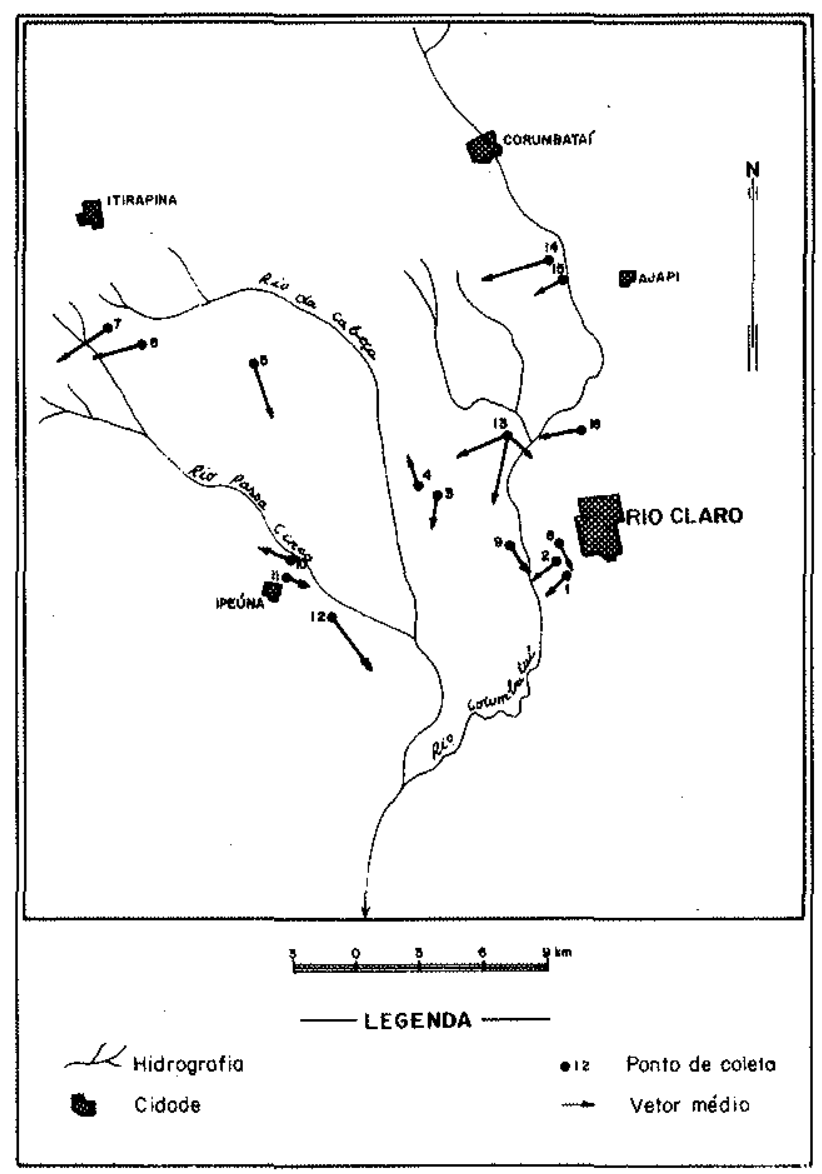

Figura 1 - Vetores médios encontrados a partir do eixo maior de seixos alongados de cascalheiras da Formação Rio Glaro, Bacia do Rio Corumbataí (SP)

Figura 2 - Área coberta pelos sedimentos da Formação Rio Cláro. As linhas interrompidas indicam o sentido geral de transporte, segundo Björnberg e Landim (1966)

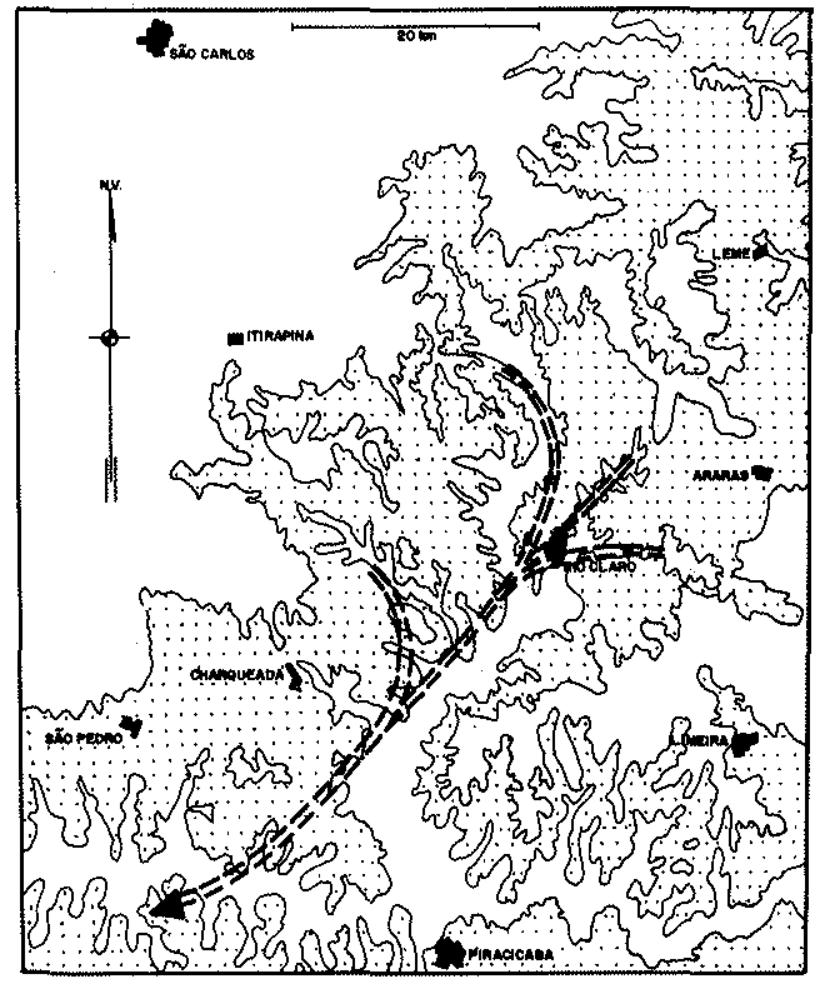


BIBLIOGRAFIA

ALLEN, J. R. L. - 1970 - Physical Processes of Sedimentation: American Elsevier Publ.

BJÖRNBERG, A. J. S. - 1965 - Sedimentos pós-cretáceos do leste do Estado de São Paulo. Tese para concurso de livre docência, Escola de Engenharia de São Carlos, USP

BJÖRNBERG, A.J.S. e LANDIM, P. M. B. - 1966 - Contribuição ao estudo da Formação Rio Claro (Neocenozóico). Bol. Soc. Bras. Geol., 15 (4): 43-67

BJÖRNBERG, A. J.S., LANDIM, P. M. B. e MEIRELLES F. ${ }^{\circ}$, G. M. - 1964 - Restos de plantas modernas em niveis elevados na região de Rio Claro. Escola de Engenharia de São Carlos, Geologia (11): $37-57$

FÚLFARO, V. J. e SUGUIO, K. - 1968 - A Formação Rio Claro (Neocenozóico) e seu ambiente de deposição. I.G.G., 20: 45-60

GREENWOOD, J.A. and DURAND, D. - 1955 - The distribution of Length and components of the sum of random unit vectores. Ann. Math. Stat., 26: 233-246

JOHANSSON, C. E. - 1965 - Structural studies of sedimentary deposits. Geol. Fören-Förhandlingar, 87: 3-61

JONES, THOMAS A. - 1968 - Statistical Analysis of Orientation Data: Jour. Sed. Petrology, $38(1): 61-67$

KÜRSTEN, M. - 1960 - Zur Frage der Geröllorientierung un Flussläuten. Geol. Rundschau, 49 : 498-501

LANDIM, P. M.B., MEYER, Jr., A. E. e PEREIRA, N. A. M. - 1974 - Determinação de paleocorrentes pelo estudo da orientação de seixos. A ser publicado em Not. Geomorfológica

MEZZALIRA, S. - 1964 - Novas Ocorrências de vegetais fósseis cenozóicos no Estado de São Paulo. Rev. O I.G.G., 15: 73-94

MISES, R. V. - 1918 - Uber die Ganzzahligkeit der Atomgewichte und Verwandte Fragen. Physikal. Zeitschrift, 19: 490-500

PENTEADO, M. M. - 1968 - Geomorfologia do setor centro-ocidental da depressão periférica paulista. Tese de Doutoramento, FFCL de Rio Claro

PENTEADO, M. M. - 1969 - Novas informações a respeito dos pavimentos detríticos (stone lines). Notícias Geomorfológicas, 9 (17): 15-41

PINCUS, H.J, - 1956 - Some Vector and Arithmetic Operations on Two-Dimensional Orientation Variates, With Aplications to Geologycal Data. Journal of Geology, 64 (6): 533-557

POTTER, E.P. and PETTIJOHN, F.L. - 1963 - Paleocurrents and Basin Analysis. Springer Verlag

REICHE, P. - 1938 - An Analysis of Cross-lamination: the Coconino Sandstone. Jour. Geology, 46: $905-932$

SPALLETTI, L. A. - 1972 - Sedimentologia de los Cenoglomerados de Volcan, Província de Jujuv, Argentina. Revista do Museu de La Prata, tomo VIII, Geologia, 66: 137-225

STEINMTS, R. - 1962 - Analysis of Vectorial Data. Journal of Sed. Petrology, 32 (4): $801-812$

SUGUIO, K. - 1973a - Formação Bauru, calcários e sedimentos detríticos associados. Tese de livre-docência, Inst. Geociências, USP

SUGUIO, K. - 1973b - Introdução à sedimentologia. Editora Edgard Blücher Ltda. 